\title{
On adaptive Haar approximations of random flows
}

\author{
Yuri K.Dem'yanovich, \\ Institute Saint Petersburg State University, \\ St. Petersburg, 198504 \\ Russia
}

Received: May 27, 2020. Revised: January 7, 2021. Accepted: February 2, 2021. Published: February 11, 2021.

\begin{abstract}
The adaptive approximations for some characteristic of random functions defined on arbitrary irregular grids are discussed in this paper. The mentioned functions can be examined as flows of random real values associated with an irregular grid. This paper considers the question of choosing an adaptive enlargement of the initial grid. The mentioned enlargement essentially depends on the formulation of the criterion in relation to which adaptability is considered. Several criteria are considered here, among which there are several criteria applicable to the processing of random flows. In particular, the criteria corresponding to the mathematical expectation, dispersion, as well as autocorrelation and cross-correlation of two random flows are considered. It is possible to consider criteria corresponding to various combinations of the mentioned characteristics. The number of knots of the initial (generally speaking, irregular) grid can be arbitrary, and the main grid can be any subset of the initial one. Decomposition algorithms are proposed, taking into account the nature of the changes in the initial flow. The number of arithmetic operations in the proposed algorithms is proportional to the length of the initial flow. Sequential processing of the initial flow is possible in real time.
\end{abstract}

Keywords- random functions, Haar-type basis, adaptive approximation

\section{INTRODUCTION}

$\mathrm{T}$ HE Numerical flows of random variables are often associated with the uniform grid. In this case, they are called time series. Time series are widely used in various fields, from economics to geological and astronomical research. A large number of time series are dedicated to research (a detailed bibliography can be found in the works 1 - 22 $)$.

Modern research significantly deepens the previously obtained results. The swarming principles of insect life lead to the creation new algorithms, for example, to develop an optimal IIR filter (see [11]). Various forms of approximation are supported by the evolving apparatus of cubic splines (see [12]), Haar-type splines etc. New ways to predict the history of processes in high-performance computing systems [13] are associated with processes that can be considered like as random processes. Since in the discrete systems also found elements of chaos [14, here the statistical methods can be very helpful. Continue statistical studies of block-chain structures in connection with the problem of increasing their reliability and the stability of [15].

When a random process is sampled, the resulting sampling sequence will depend on the specific implementation of the discretized process. In many problems it is required to consider finite in time fragment of a random process with length $N$ samples. In this case the correlation function can be represented as a correlation matrix (it is assumed that the random process has zero mathematical expectation) $(\overline{[x(i), x(j)]})_{i, j \in\{0,1, \ldots, N\}}$, where the bar above denotes averaging over the ensemble of realizations. If the process is nonstationary, all elements of the matrix can be different. In the case of a stationary process, the correlation matrix is completely determined by its first row and first column, since along all diagonals parallel to the main one, there are the same elements.

In practice, it is very often required to switch from analog signal to discrete. This transition is connected with sampling and digitization processes (the latter is also called quantization). Such a transition is determined by the capabilities of digital computing. A computer can only represent a finite quantity of numbers. The other numbers are presented with an error called rounding error. Usually this error is considered as a random variable. Analysis distribution of this random variable is very complicated, its characteristics depend on the properties of the computing system. However, in most cases there is sufficient reason to consider this random variable uniformly distributed in some interval (see 22]). Note that non-uniform quantization is used in practice. The idea of the nonuniform quantization in the general cases is formulated as follows: the range of possible signal values is divided by $N$ quantization zones $a_{0} \ldots a_{1}, a_{1} \ldots a_{2}, \ldots, a_{N-1} \ldots a_{N}$, and the $i$-th zone is mapped to the quantization value $b_{i}$. The ranges are determined by the probability density of the signal falling into the appropriate range. If the probability density is not known, the ranges are determined by an instance of the "most typical" signal. Uneven quantization is widely used. It used, for example, in modern digital telephone networks. Small values of the speech signal more likely 
than large, so a non-linear signal conversion: the range of values is represented by the 12-th discharges (4096 levels of uniform quantization) at non-uniform quantization is quantized by 256 (total 8 ranges) of unevenly spaced levels. However, other schemes are also possible.

Thus, the transmitted information contains elements of randomness and is a discrete random process. Transfer of their numerical characteristics by communication lines can be difficult due to the large volume of information.

It is possible that rapidly changing data will have to be transmitted completely. Slowly changing data can be transferred only rarely because the restoration of the information is very simple in this case. This will significantly reduce the load on transceiver devices and communication lines. For efficiency the exchange of information requires that the sender and the recipient use the agreed means of data processing. One of the options for such means is the adaptive Haar's approximation proposed in this work. We consider the approximation of the mathematical expectation, variance and correlation matrix or their estimations calculated over the set of realizations.

The purpose of this work is to consider the numerical flows of characteristics for random quantity associated with a (generally speaking) non-uniform grid, and to investigate the properties of their adaptive approximation by the Haar spaces. As a result the getting flows (so called main flows) of probabilistic characteristics have a smaller volume than the initial one. In addition, the obtained main flows contain significantly less elements than the initial ones. The usage of the main flow allows us to obtain the main trend of the initial flow in compact form.

The resulting main flow is the first step in constructing an adaptive wavelet decomposition. The classical approach (see [7] - [10]) uses a two-fold coarsening of the grid (by removing odd knots). It is very difficult to get adaptability along this path. The non-classical approach (see [16 - 21]) applied in this work allows the use of an arbitrary non-uniform grid and the enlargement of it by removing an arbitrary set of knots. With this approach the construction of fragments of fast changes are associated with a fine grid. The fragments of slow changes are associated with a coarse grid. As a result the main flow is a qualitative approximation of the initial flow.

The adaptive approximations for some characteristic of random functions defined on arbitrary irregular grids are discussed in this paper. The mentioned functions can be examined as flows of random real values associated with an irregular grid. This paper considers the question of choosing an adaptive enlargement of the initial grid. The mentioned enlargement essentially depends on the formulation of the criterion in relation to which adaptability is considered. Several criteria are considered here, among which there are several criteria applicable to the processing of random flows. In particular, the criteria corresponding to the mathematical expectation, dispersion, as well as autocorrelation and cross-correlation of two random flows are considered. It is possible to con- sider the criteria corresponding to various combinations of the mentioned characteristics. The number of knots of the initial (generally speaking, irregular) grid can be arbitrary, and the main grid can be any subset of the initial one. Decomposition algorithms are proposed, taking into account the nature of the changes in the initial flow. The number of arithmetic operations in the proposed algorithms is proportional to the length of the initial flow. Sequential processing of the initial flow is possible in real time.

\section{EMBEDDED GRID}

On the interval $(\alpha, \beta)$ of the real axis $\mathbf{R}^{1}$ consider the grid

$$
\Xi: \ldots<\xi_{-2}<\xi_{-1}<\xi_{0}<\xi_{1}<\xi_{2} \ldots
$$

with properties

$$
\lim _{\text {to- }-\infty} \xi_{j}=\alpha, \quad \lim _{j \rightarrow+\infty} \xi_{j}=\beta .
$$

If $c \in \Xi$, then there exists $i \in \mathbf{Z}$ such that $c=\xi_{i}$; in this case we denote $c^{-}=\xi_{i-1}, c^{+}=\xi_{i+1}$.

We suppose that $c, d \in \Xi, \quad c^{+}<d^{-}$, i.e. for some $i, j \in \mathbf{Z}, i+2<j$, the equalities $c=\xi_{i}, d=\xi_{j}$ are true. We will use the notation $|[c, d]|=\left\{\xi_{s} \mid c \leq \xi_{s} \leq d, s \in\right.$ $\mathbf{Z}\}, \mid\left[c, d \mid\left[=\left\{\xi_{s} \mid c \leq \xi_{s}<d, s \in \mathbf{Z}\right\}\right.\right.$. The set $|[c, d]|$ is called a grid segment, and the set $\mid[c, d \|[$ is called a grid interval. It's clear that $\mid\left[c, d \mid\left[=\left|\left[c, d^{-}\right]\right|\right.\right.$.

The function defined on the grid $\Xi$ is named a discrete function. The set of discrete functions $u(t)$ defined on the grid $\Xi$ is denoted $C(\Xi)$; it is clear that $C(\Xi)$ is a linear space.

Consider also the linear normed space $C|[c, d]|$ of functions $u(t)$ defined on $|[c, d]|$; here the norm is introduced by the ratio

$$
\|u\|_{C|[c, d]|}=\max _{t \in|[c, d]|}|u(t)| .
$$

In a similar way, we introduce the normed linear space $C \mid[c, d \mid[$ functions $u(t)$, defined on $\mid[c, d \mid[$, with the norm

$$
\|u\|_{C \mid[c, d \mid[}=\max _{t \in \mid[c, d \mid[}|u(t)| .
$$

Obviously, the spaces $C|[c, d]|$ and $C \mid[c, d \mid[$ are finite-dimensional.

Let us denote $J_{m}=\{0,1,2, \ldots, m\}$. Let $M$ be a natural number $M>2$ and $|[a, b]|=\left\{\xi_{s}\right\}_{s \in J_{M+1}}$, so that

$$
|[a, b]|=\left\{a=\xi_{0}<\xi_{1}<\ldots<\xi_{M}<\xi_{M+1}\right\} .
$$

A value $\tau=\max _{j \in J_{M}}\left(\xi_{j+1}-\xi_{j}\right)$ is called the maximum step in the grid segment (3). The set of grid segments, contained in the grid segment $|[a, b]|$, denote $\mathcal{O}$, so as

$$
\mathcal{O}=\mathcal{O}(|[a, b]|)=\{|[c, d]|||[c, d]|\subset|[a, b] \mid\} .
$$

Let $\mathcal{M}$ be some grid segment from the set $\mathcal{O}$. Consider the mapping $f: \quad \mathcal{M} \mapsto \mathbf{R}^{\mathbf{1}}$, having the following properties.

$$
f(\mathcal{M}) \geq 0 \forall \mathcal{M} \subset \mathcal{O}, \quad f(|[c, c]|)=0 \forall c \in|[a, b]|,
$$




$$
\mathcal{M}^{\prime} \subset \mathcal{M}^{\prime \prime} \Longrightarrow f\left(\mathcal{M}^{\prime}\right) \leq f\left(\mathcal{M}^{\prime \prime}\right) \quad \forall \mathcal{M}^{\prime}, \mathcal{M}^{\prime \prime} \subset \mathcal{O}
$$

The mapping $f$ with the indicated properties will be called pseudo-measure on the grid segment |[a,b]| .

Remark 1. Let $f_{0}$ be the function of sets, defined on collection

$$
\mathcal{O}_{0}=\mathcal{O}_{0}([a, b])=\{[c, d] \mid[c, d] \subset[a, b]\} .
$$

If $f_{0}$ satisfies the conditions

$$
\begin{gathered}
f_{0}(\mathcal{N}) \geq 0 \forall \mathcal{N} \subset \mathcal{O}_{0}, \quad f_{0}([c, c])=0 \forall c \in[a, b], \\
\mathcal{N}^{\prime} \subset \mathcal{N}^{\prime \prime} \Longrightarrow f_{0}\left(\mathcal{N}^{\prime}\right) \leq f_{0}\left(\mathcal{N}^{\prime \prime}\right) \quad \forall \mathcal{N}^{\prime}, \mathcal{N}^{\prime \prime} \subset \mathcal{O}_{0},
\end{gathered}
$$

then the restriction $f$ of the function $f_{0}$ on the collection $\mathcal{O}$ is the pseudo-measure on the grid segment $|[a, b]|$.

Let be

$$
\varepsilon \in\left(\varepsilon^{*}, \varepsilon^{* *}\right)
$$

where

$$
\varepsilon^{*}=\max _{\xi \in\left|\left[a, b^{-}\right]\right|} f\left(\left\{\xi, \xi^{+}\right\}\right), \quad \varepsilon^{* *}=f(|[a, b]|) .
$$

Consider the grid $X \subset \|[a, b] \mid$,

$$
\begin{gathered}
X=X(f, \varepsilon,|[a, b]|): \\
a=x_{0}<x_{1}<\ldots<x_{K}<x_{K+1}=b
\end{gathered}
$$

such that

$$
\begin{gathered}
f\left(\mid\left[x_{s}, x_{s+1}\right) \leq \varepsilon<f\left(x_{s}, x_{s+1}^{+}\right)\right. \\
\forall s \in\{0,1, \ldots, K-1\}, \\
f\left(x_{K}, b\right) \leq \varepsilon, \quad X \subset|[a, b]| .
\end{gathered}
$$

Definition 1. Grid (8) with properties (9) - (10) is called an adaptive grid (for pseudo-measure $f$ on grid segment $|[a, b]|)$ or, for short, a $f$-adaptive grid.

The following statement is true

Theorem 1. If the relations (4) - (7) are satisfied, then a natural number $K=K(f, \varepsilon,|[a, b]|)$ and a grid (8) with properties (9) - (10) exist.

Proof is carried out by the mathematical induction.

Obviously, the integer function $K(f, \varepsilon,|[a, b]|)$ has monotonicity property: if $\varepsilon^{*}<\varepsilon^{\prime} \leq \varepsilon^{\prime \prime}<\varepsilon^{* *}$, then $K\left(f, \varepsilon^{\prime},|[a, b]|\right) \geq K\left(f, \varepsilon^{\prime \prime},|[a, b]|\right)$.

Summing the relations (9), we obtain the inequality

$$
\begin{gathered}
\sum_{s=0}^{K-1} f\left(\left|\left[x_{s}, x_{s+1}\right]\right|\right) \leq \varepsilon K(f, \varepsilon,|[a, b]|)< \\
<\sum_{s=0}^{K-1} f\left(\left|\left[x_{s}, x_{s+1}^{+}\right]\right|\right) .
\end{gathered}
$$
$K$.

The inequality (11) gives the bounds for the number

\section{Computational Complexity of the ADAPTIVE GRID}

It is easy to see that the proof of Theorem 1 contains the algorithm constructing an adaptive grid $X$. Consider the question of the complexity of this algorithm.

Let $N(f)$ and $N_{\text {comp }}=N_{\text {comp }}(f)$ be a number of calculations of pseudo-measures $f$ and the number of comparisons in the considered algorithm 1 .

Theorem 2. The algorithm for constructing an adaptive grid $X$ has the following properties

$$
N(f)=N_{\text {comp }}(f)=K+M+2 .
$$

Proof. The adaptive grid can be written in form $X=X(f, \varepsilon,|[a, b]|)$ :

$$
a=x_{0}=\xi_{0}<x_{1}=\xi_{p_{1}}<\ldots
$$

$$
\ldots<x_{K}=\xi_{p_{K}}<x_{K+1}=\xi_{p_{K+1}}=b,
$$

where $p_{K+1}=M+1$. At the $s$ th step of this algorithm, we go from knot $x_{s}$ to knot $x_{s+1}$.

Suppose $x_{s}=\xi_{p_{s}}, x_{s+1}=\xi_{p_{s+1}}$. It is not difficult to see that the mentioned transition requires

1 ) to calculate $p_{s+1}-p_{s}+1$ times the pseudo-measure $f$ (on grid segments $\left|\left[\xi_{p_{s}}, \xi_{p_{s}+1}\right]\right|,\left|\left[\xi_{p_{s}}, \xi_{p_{s}+2}\right]\right|, \ldots$, $\left.\left|\left[\xi_{p_{s}}, \xi_{p_{s+1}+1}\right]\right|\right)$

2) to compare the result with $\varepsilon$ follows $p_{s+1}-p_{s}+1$ times.

Since $s$ changes from 0 to $K$, then the total number $N(f)$ of calculations of the pseudo-measure $f$ is

$$
N(f)=\sum_{s=0}^{K}\left(p_{s+1}-p_{s}+1\right)=K+1+p_{K+1}-p_{0} .
$$

According to the formulas (8) and (13), $p_{0}=0, p_{K+1}=$ $M+1$, so as a result we get $N(f)=K+M+2$. In the same way we find the number of $N_{\text {comp }}$ comparisons. So the formulas (12) are valid. This ends the proof.

\section{An Auxiliary Evaluations}

Suppose that the function $u(t)$ is given on the grid segment

$$
|[a, b]|: \quad a=\xi_{0}<\xi_{1}<\ldots<\xi_{M}<\xi_{M+1}=b .
$$

Let us discuss

$$
a \leq y<t \leq b, \quad y, t \in|[a, b]|, \quad y=\xi_{i}, \quad t=\xi_{j} .
$$

We use a notion

$$
\mathcal{D}_{\Xi} u(\xi)=\frac{u\left(\xi^{+}\right)-u(\xi)}{\xi^{+}-\xi} .
$$

In the case when the function $u$ depends on two variables, $u=u(t, z), u(\bullet, z) \in C|[a, b]|, z \in|[a, b]|$,

\footnotetext{
${ }^{1}$ Implementation of the listed operations includes operations assignments to one or another auxiliary variable. Separated accounting of assignment operations are not performed in this work.
} 
let us agree to always use the operator $D_{\Xi}$ only of the first of the two variables,

$$
\mathcal{D}_{\Xi} u(\xi, z)=\frac{u\left(\xi^{+}, z\right)-u(\xi, z)}{\xi^{+}-\xi} .
$$

From (14) - (17) we get

$$
u(t)-u(y)=\sum_{s=i}^{j-1} \frac{u\left(\xi_{s+1}\right)-u\left(\xi_{s}\right)}{\xi_{s+1}-\xi_{s}}\left(\xi_{s+1}-\xi_{s}\right) .
$$

Lemma 1. For a discrete function $u \in C|[a, b]|$ the inequality holds

$$
\begin{gathered}
|u(t)-u(y)| \leq \max _{\xi \in\left|\left[y, t^{-}\right]\right|}\left|D_{\Xi} u(\xi)\right|(t-y) \\
y<t, \quad y, t \in|[a, b]| .
\end{gathered}
$$

If $u \in C^{1}[a, b]$ then

$$
|u(t)-u(y)| \leq \max _{\xi \in[y, t]}\left|u^{\prime}(\xi)\right|(t-y) .
$$

Proof. Consider the relation (18), assuming $y=\xi_{i}$ and $t=\xi_{j}$. Taking into account the positivity of the differences $\xi_{s+1}-\xi_{s}, t-y$ and notation (16), we see that the formulas $(19)-(20)$ follow directly from the relation (18). Formula (21) follows from the Lagrange theorem.

\section{Generalized HaARs' Functions}

We introduce piecewise constant functions $\omega_{j}(t), t \in$ $(\alpha, \beta)$ defined by equalities

$$
\omega_{j}(t)= \begin{cases}1 & \text { for } \quad t \in\left[\xi_{j}, \xi_{j+1}\right) \\ 0 & \text { for } \quad t \in \mathbf{R}^{\mathbf{1}} \backslash\left[\xi_{\mathbf{j}}, \xi_{\mathbf{j}+\mathbf{1}}\right) .\end{cases}
$$

System $\left\{\omega_{j}(t)\right\}_{j \in \mathbf{Z}}$ of functions (22) is defined by the grid (1) - (2) . It is a linearly independent system.

The functions $\omega_{j}(t)$ are called coordinate splines of order zero..$^{2}$ Let $S_{j}=\left[\xi_{j}, \xi_{j+1}\right]$. It's clear that $\operatorname{supp} \omega_{j}=S_{j}$. The approximation relation $\sum_{j \in \mathbf{Z}} \omega_{J}(t) \equiv$ $1 \forall t \in(\alpha, \beta)$ in this case is the decomposition of unity into interval $(\alpha, \beta)$.

For fixed $t \in(\alpha, \beta)$ the linear combination

$$
u(t)=\sum_{j \in \mathbf{Z}} c_{j} \omega_{j}(t)
$$

consists of one term: for $t \in\left[\xi_{i}, \xi_{i+1}\right)$ formula (23) takes the form $u(t)=c_{i} \omega_{i}(t)=c_{i}$.

Linear space $\mathbf{S}_{0}(\Xi)$, defined by the relation

$$
\mathbf{S}_{0}(\Xi)=C l_{p}\left\{u(t) \mid u(t)=\sum_{j} c_{j} \omega_{j} \quad \forall c_{j} \in \mathbf{R}^{1}\right\},
$$

is called the generalized Haar space on the grid $\Xi$, and the elements of this space are splines of zero order. Symbol $C l_{p}$ in (24) denotes closure in the topology of the pointwise convergence. Specifying the grid $\Xi$ uniquely determines the space $\mathbf{S}_{0}(\Xi)$.

The next point is about building embedded space splines of the order zero on an embedded grid.

\footnotetext{
${ }^{2}$ In the case when the grid $\Xi$ is uniform (and therefore $\alpha=-\infty$, $\beta=+\infty)$, the functions $\omega_{j}$ defined above are often called Haar functions.
}

\section{Approximation By the HaAR type FUNCTIONS}

Consider a grid $\widehat{X}$, which is a subset of the grid $\Xi$

$$
\widehat{X}: a=\widehat{x}_{0}<\ldots<\widehat{x}_{\widehat{K}}<\widehat{x}_{\widehat{K}+1}=b, \quad \widehat{X} \subset \Xi .
$$

We discuss an interpolate operator $H_{\widehat{X}}$ of the Haar type such that for the grid function $u(t), u \in C|[a, b]|$, we have the piecewise constant interpolation $H_{\widehat{X}} u$

$$
\begin{gathered}
\left(H_{\widehat{X}} u\right)(t)=u\left(\widehat{x}_{j}\right) \quad \forall t \in\left[\widehat{x}_{j}, \widehat{x}_{j+1}\right), \\
j \in\{0,1, \ldots, \widehat{K}\} .
\end{gathered}
$$

In the case when the function $u$ depends on two variables, $u=u(t, z), u(\bullet, z) \in C|[a, b]|, z \in|[a, b]|$, let's agree to always use the operator $H_{\widehat{X}}$ only on the first of the two variables,

$$
\left(H_{\widehat{X}} u\right)(t, z)=u\left(\widehat{x}_{j}, z\right) \quad \forall t \in\left[\widehat{x}_{j}, \widehat{x}_{j+1}\right) .
$$

Theorem 3. If $u \in C|[a, b]|$, then for $t \in$ $\mid\left[\widehat{x}_{j}, \widehat{x}_{j+1} \mid[\right.$ the inequality holds

$$
\begin{aligned}
\mid u(t) & -\left(H_{\widehat{X}} u\right)(t) \mid \leq\left(\widehat{x}_{j+1}-\widehat{x}_{j}\right) \times \\
& \times \max _{\left.\xi \in \mid \widehat{x}_{j}, \widehat{x}_{j+1}^{-}\right] \mid}\left|\mathcal{D}_{\Xi} u(\xi)\right| .
\end{aligned}
$$

If $u \in C^{1}[a, b]$ then

$$
\left|u(t)-\left(H_{\widehat{X}} u\right)(t)\right| \leq \max _{\xi \in\left[\widehat{x}_{j}, \widehat{x}_{j+1}\right]}\left|u_{t}^{\prime}(\xi)\right|\left(\widehat{x}_{j+1}-\widehat{x}_{j}\right) .
$$

Proof. Taking into account notation $(26)-(27)$ we put $y=\hat{x}_{j}, t \in\left|\left[\widehat{x}_{j}, \widehat{x}_{j+1}^{-}\right]\right|$in formula (19):

$$
\begin{gathered}
\left|u(t)-\left(H_{\widehat{X}} u\right)(t)\right|=\left|u(t)-u\left(\widehat{x}_{j}\right)\right|=|u(t)-u(y)| \leq \\
\leq \max _{\xi \in\left|\left[y, t^{-}\right]\right|}\left|\mathcal{D}_{\Xi} u(\xi)\right|(t-y) .
\end{gathered}
$$

To prove the inequality (28) it remains to return to the initial notation in the relation $(30)$ and take advantage of the inequality $t<\widehat{x}_{j+1}$. Inequality (29) follows from the Lagrange formula.

\section{EVALUATIONS FOR RANDOM FUNCTIONS}

In this section, we consider random flows and random functions.

Let $v(t)$ and $w(t)$ be a random functions defined on the grid segment

$$
\mid\left[a, b \mid\left[: \quad a=\xi_{0}<\xi_{1}<\ldots<\xi_{M}<\xi_{M+1}=b,\right.\right.
$$

or on the usual segment $[a, b]$ of the real axis $\mathbf{R}^{\mathbf{1}}$. In the first case we talk about a discrete random function, in the second case we talk about a nondiscrete random function. Consider $t, t^{\prime} \in \mid\left[a, b \mid\left[\right.\right.$ (or $t, t^{\prime} \in[a, b]$ accordingly). We suppose that the mathematical expectation $\mathbf{M}[v](t)$, the desperation $\mathbf{D}[v](t)$, and correlations $\left[v(t), v\left(t^{\prime}\right)\right],\left[v(t), w\left(t^{\prime}\right)\right]$ of their $t, t^{\prime}$-sections exist. 
For centered random functions, we will supply "zero" above; e.g., $v^{0}(t)=v(t)-\mathrm{M}[v](t)$. For centered random functions the correlation function possesses the properties of a bilinear form.

Using Lemma 1 for different functions $u(t)$ we deduce important assertions for nonrandom characteristics of the random functions $v(t)$ and $w(t)$.

If we use Lemma 1 for $u(t)=\left[v^{0}(t), w^{0}(y)\right]$, then we obtain the next statement.

Theorem 4. For a centered discrete random functions $v^{0}, w^{0} \in C \mid[\mathrm{a}, \mathrm{b} \mid[$ the inequalities

$$
\begin{aligned}
& \left|\left[v^{0}(t), w^{0}\left(t^{\prime}\right)\right]-\left[v^{0}(y), w^{0}\left(t^{\prime}\right)\right]\right| \leq \\
& \leq \max _{\xi \in \mid\left[y, t^{-} \mid[\right.}\left|\mathcal{D}_{\Xi}\left[v^{0}(\xi), w^{0}\left(t^{\prime}\right)\right]\right|(t-y), \\
& a \leq y<t<b, \quad y, t, t^{\prime} \in \mid\left[a, b^{-} \mid[,\right.
\end{aligned}
$$

are right. If centered nondiscrete random functions $v^{0}, w^{0}$ such that the function $\varphi(t)=\left[v^{0}(t), w^{0}\left(t^{\prime}\right)\right]$ has the property $\varphi \in C^{1}[a, b] t^{\prime} \in[a, b]$, then the inequalities

$$
\begin{aligned}
& \left|\left[v^{0}(t), w^{0}\left(t^{\prime}\right)\right]-\left[v^{0}(y), w^{0}\left(t^{\prime}\right)\right]\right| \leq \\
& \leq \max _{\xi \in[t, y]}\left|\left[v^{0}(\xi), w^{0}\left(t^{\prime}\right)\right]_{\xi}^{\prime}\right|(t-y), \\
& a \leq y \leq t<b, \quad y, t, t^{\prime} \in[a, b]
\end{aligned}
$$

are fulfilled.

Corollary 1. For a centered discrete random function $v^{0} \in C \mid[a, b \mid[$ the inequalities

$$
\begin{gathered}
\left.\mid\left[v^{0}(t), v^{0}(y)\right]-\mathbf{D}\left[v^{0}\right](y)\right] \mid \leq \\
\leq \max _{\xi \in \mid\left[y, t^{-} \mid[\right.}\left|\mathcal{D}_{\Xi}\left[v^{0}(\xi), v^{0}(y)\right]\right|(t-y), \\
a \leq y \leq t<b, \quad y, t \in \Xi
\end{gathered}
$$

are right. A centered nondiscrete random function $v^{0}$ such that the function $\varphi(t)=\left[v^{0}(t), v^{0}(y)\right], y \in[a, b]$, has the property $\varphi \in C[a, b]$, the inequalities

$$
\begin{gathered}
\left|\left[v^{0}(t), v^{0}(y)\right]-\mathbf{D}\left[v^{0}\right](y)\right| \leq \\
\leq \max _{\xi \in[t, y]}\left|\left[v^{0}(\xi), v^{0}(y)\right]_{\xi}^{\prime}\right|(t-y), \\
a \leq y \leq t<b, \quad y, t \in[a, b]
\end{gathered}
$$

are fulfilled.

Proof. Formulas $(36)-(39)$ follow from relations (32) $-(35)$, where $w^{0}=v^{0}$ and $t^{\prime}=y$.

Theorem 5. For a discrete random function $v \in$ $C \mid[a, b \mid[$ the inequalities

$$
\begin{aligned}
& |\mathbf{M}[v](t)-\mathbf{M}[v](y)| \leq \max _{\xi \in \mid\left[y, t^{-} \mid[\right.}\left|\mathcal{D}_{\Xi} \mathbf{M}[v](\xi)\right|(t-y), \\
& |\mathbf{D}[v](t)-\mathbf{D}[v](y)| \leq \max _{\xi \in \mid\left[y, t^{-} \mid[\right.}\left|\mathcal{D}_{\Xi} \mathbf{D}[v](\xi)\right|(t-y),
\end{aligned}
$$

$$
a \leq y \leq t<b
$$

are right. If a nondiscrete random function $v$ such as the function $\varphi(t)=\mathbf{M}[v](t)$ has the property $\varphi \in C^{1}[a, b]$, then the inequality

$$
|\mathbf{M}[v](t)-\mathbf{M}[v](y)| \leq \max _{\xi \in[y, t]}\left|(\mathbf{M}[v](\xi))_{\xi}^{\prime}\right|(t-y),
$$

is fulfilled. If a nondiscrete random function $v$ such as the function $\varphi_{1}(t)=\mathbf{D}[v](t)$ has the property $\varphi_{1} \in$ $C^{1}[a, b]$, then the inequality

$$
\begin{gathered}
|\mathbf{D}[v](t)-\mathbf{D}[v](y)| \leq \max _{\xi \in[y, t]}\left|(\mathbf{D}[v](\xi))_{\xi}^{\prime}\right|(t-y), \\
a \leq y \leq t<b
\end{gathered}
$$

is right.

Proof. Using Lemma 1 for $u=\mathbf{M}[v]$ and for $u=$ $\mathbf{D}[u]$, we obtain the relations $(40)-(45)$ respectively.

\section{ApProximations FOR RANDOM FUnCTIONS}

Consider grid (25) and approximation (26) in connection with the characteristics of random functions.

Theorem 6. For centered discrete random functions $v^{0}, w^{0} \in C \mid[a, b \mid[$ the inequalities

$$
\begin{gathered}
\left|\left[v^{0}(t), w^{0}\left(t^{\prime}\right)\right]-\left[v^{0}\left(\widehat{x}_{j}\right), w^{0}\left(t^{\prime}\right)\right]\right| \leq \\
\leq \max _{\xi \in\left|\widehat{x}_{j}, \widehat{x}_{j+1}^{-}\right|[}\left|\mathcal{D}_{\Xi}\left[v^{0}(\xi), w^{0}\left(t^{\prime}\right)\right]\right|\left(t-\widehat{x}_{j}\right), \\
a \leq \widehat{x}_{j} \leq t<\widehat{x}_{j+1}, \quad t^{\prime} \in-[\mathrm{a}, \mathrm{b}-[.
\end{gathered}
$$

are right. For centered nondiscrete random functions $v^{0}, w^{0}$ such as the function $\varphi(t)=\left[v^{0}(t), w^{0}\left(t^{\prime}\right)\right]$ has the property $\varphi \in C^{1}[a, b]$, the inequalities

$$
\begin{aligned}
& \left|\left[v^{0}(t), w^{0}\left(t^{\prime}\right)\right]-\left[v^{0}\left(\widehat{x}_{j}\right), w^{0}\left(t^{\prime}\right)\right]\right| \leq \\
& \leq \max _{\xi \in\left[t, \widehat{x}_{j}\right]}\left|\left[v^{0}(\xi), w^{0}\left(t^{\prime}\right)\right]_{\xi}^{\prime}\right|\left(t-\widehat{x}_{j}\right), \\
& a \leq \widehat{x}_{j} \leq t<\widehat{x}_{j+1}, \quad t^{\prime} \in[a, b]
\end{aligned}
$$

are fulfilled.

Corollary 2. For the centered discrete random function $v^{0} \in C \mid[a, b \mid[$ the inequalities

$$
\begin{gathered}
\left|\left[v^{0}(t), v^{0}\left(\widehat{x}_{j}\right)\right]-\mathbf{D}\left[v^{0}\right]\left(\widehat{x}_{j}\right)\right| \leq \\
\leq \max _{\xi \in\left|\widehat{x}_{j}, \widehat{x}_{j+1}^{-}\right|[}\left|\mathcal{D}_{\Xi}\left[v^{0}(\xi), v^{0}\left(\widehat{x}_{j}\right)\right]\right|\left(t-\widehat{x}_{j}\right), \\
a \leq \widehat{x}_{j} \leq t<\widehat{x}_{j+1}, \quad t \in \Xi .
\end{gathered}
$$

are fulfilled. For a centered nondiscrete random function $v^{0}$ such as the function $\varphi(t)=\left[v^{0}(t), v^{0}(y)\right]$ has the property $\varphi \in C[a, b]$, the inequalities

$$
\left|\left[v^{0}(t), v^{0}\left(\widehat{x}_{j}\right)\right]-\mathbf{D}\left[v^{0}\right]\left(\widehat{x}_{j}\right)\right| \leq
$$




$$
\begin{aligned}
& \leq \max _{\xi \in\left[t, \widehat{x}_{j}\right]}\left|\left[v^{0}(\xi), v^{0}\left(\widehat{x}_{j}\right)\right]_{\xi}^{\prime}\right|\left(t-\widehat{x}_{j}\right), \\
& a \leq \widehat{x}_{j} \leq t<\widehat{x}_{j+1}, \quad t \in[a, b],
\end{aligned}
$$

are right.

Proof. Formulas (50) - (53) follow from Theorem 6, if we take $w^{0}=v^{0}$ and $t^{\prime}=\widehat{x}_{j}$ in (46) $-(49)$.

Theorem 7. For a discrete random function $v \in$ $C \mid[a, b \mid[$ the inequalities

$$
\begin{gathered}
\left|\mathbf{M}[v](t)-\mathbf{M}[v]\left(\widehat{x}_{j}\right)\right| \leq \\
\leq \max _{\xi \in\left|\widehat{x}_{j}, \widehat{x}_{j+1}^{-}\right|[}\left|\mathcal{D}_{\Xi} \mathbf{M}[v](\xi)\right|\left(t-\widehat{x}_{j}\right), \\
\left|\mathbf{D}[v](t)-\mathbf{D}[v]\left(\widehat{x}_{j}\right)\right| \leq \\
\leq \max _{\xi \in\left|\widehat{x}_{j}, \widehat{x}_{j+1}^{-}\right|[}|\mathcal{D} \Xi \mathbf{D}[v](\xi)|\left(t-\widehat{x}_{j}\right), \\
\widehat{x}_{j}<t<\widehat{x}_{j+1}
\end{gathered}
$$

are right. If a nondiscrete random function $v$ such as the function $\varphi(t)=\mathbf{M}[v](t)$ has the property $\varphi \in C^{1}[a, b]$, then the inequality

$$
\left|\mathbf{M}[v](t)-\mathbf{M}[v]\left(\widehat{x}_{j}\right)\right| \leq \max _{\xi \in\left[\widehat{x}_{j}, t\right]}\left|(\mathbf{M}[v](\xi))_{\xi}^{\prime}\right|\left(t-\widehat{x}_{j}\right),
$$

is fulfilled. If a nondiscrete random function $v$ such as the function $\varphi_{1}(t)=\mathbf{D}[v](t)$ has the property $\varphi_{1} \in$ $C^{1}[a, b]$, then the inequality

$$
\begin{gathered}
\left|\mathbf{D}[v](t)-\mathbf{D}[v]\left(\widehat{x}_{j}\right)\right| \leq \max _{\xi \in\left[\widehat{x}_{j}, t\right]}\left|(\mathbf{D}[v](\xi))_{\xi}^{\prime}\right|\left(t-\widehat{x}_{j}\right), \\
\widehat{x}_{j}<t<\widehat{x}_{j+1}
\end{gathered}
$$

is right.

Proof. We use Theorem 3. In the above theorem, we put $u(t)=\mathbf{M}[v](t)$ and from formula (28) we obtain inequality (54). Now consider $u(t)=\mathbf{D}[v](t)$ and from (28) deduce inequality (55). But if the mentioned variants of the function $u(t)$ are continuously differentiable, then we can use the inequality (29). As a result, we obtain inequalities (57) and (58). This completes the proof.

\section{ADAPTIVE GRID APPROXIMATION}

This section discusses the issue of choosing a responsive grid. The mentioned grid essentially depends on the formulation of the criterion in relation to which adaptability is considered. Several criteria are considered here, among which there are several criteria applicable to the processing of random flows. In particular, the criteria corresponding to the mathematical expectation, variance, as well as autocorrelation and cross-correlation of two random flows are considered. It is possible to consider criteria corresponding to various combinations of the mentioned characteristics.
Let $\psi(\xi)$ be a function defined on grid segment $|[a, b]|$. Consider a map $\varphi: \mathcal{M} \mapsto \mathbf{R}^{\mathbf{1}} \forall \mathcal{M} \subset \mathcal{O}$, which can be represented in the form

$$
\varphi(|[c, d]|)=\max _{\xi \in|[c, d]|}|\psi(\xi)|(d-c) .
$$

Lemma 2. Map (60) is the pseudo-measure on the grid segment $|[a, b]|$.

The proof is very simple because properties (4) - (5) are evident.

Let $|[c, d]|$ be subset of $|[a, b]|$. By definition we put

$$
\begin{gathered}
u_{1}(\xi)=\left[v^{0}(\xi), w^{0}\left(t^{\prime}\right)\right], u_{2}(\xi)=\left[v^{0}(\xi), v^{0}\left(t^{\prime}\right)\right], t^{\prime} \in|[a, b]|, \\
u_{3}(\xi)=\mathbf{M}[v](\xi), \quad u_{4}(\xi)=\mathbf{D}[v](\xi), \quad \xi \in|[a, b]| .
\end{gathered}
$$

Consider maps

$$
f_{i}(|[c, d]|)=\max _{\xi \in \mid\left[c, d^{-} \mid[\right.}\left|\mathcal{D} \Xi u_{i}(\xi)\right|(d-c), \quad i=1,2,3,4 .
$$

Theorem 8. Let $i \in\{1,2,3,4\}$ be a fixed number. Suppose that the pseudo-measure $f=f_{i}$, and real number $\varepsilon>0$ satisfies conditions (6) - (7), and the grid $\widehat{X}$ matches the grid $X\left(f_{i}, \varepsilon,|[a, b]|\right)$,

$$
\widehat{X}=X\left(f_{i}, \varepsilon,|[a, b]|\right) .
$$

Then the following statements are true.

1. The number of knots $K=K\left(f_{i}, \varepsilon,|[a, b]|\right)$ of this grid satisfies the relations

$$
\begin{gathered}
\sum_{s=0}^{K-1} f\left(\left|\left[x_{s}, x_{s+1}\right]\right|\right) / \varepsilon \leq K< \\
\quad<\sum_{s=0}^{K-1} f_{i}\left(\left|\left[x_{s}, x_{s+1}^{+}\right]\right|\right) / \varepsilon .
\end{gathered}
$$

2. The inequality is true

$$
\left|u_{i}(t)-H_{\widehat{X}} u_{i}(t)\right| \leq \varepsilon \quad \forall t \in|[a, b]| .
$$

3. The algorithm for constructing an adaptive grid $X$ has the following properties

$$
N\left(f_{i}\right)=N_{\text {comp }}\left(f_{i}\right)=(K+M+2) .
$$

Proof. Because map (60) satisfies the properties (4) - (5), formula (62) follows from the previously established relation (11). Inequality (63) follows from formulas (9) and (28). Finally, the formula (64) is obtained from (12) with the mentioned choice pseudo-measures $f$. This concludes the proof.

If $u_{i} \in C^{1}[a, b]$, then sometimes it is more suitable to discuss a measures

$$
\widetilde{f}_{i}([c, d])=\max _{\xi \in[c, d]}\left|u_{i}{ }^{\prime}(\xi)\right|(d-c), \quad i=1,2,3,4 .
$$

According to Lemma 2 and Remark 1 the maps $\widetilde{f}_{i}$ can be discussed as the pseudo-measures $\widetilde{f}_{i}(|[c, d]|)$ on 
the grid segment $|[a, b]|$. Analogously to the proof of Theorem 8 the next assertion can be obtained.

Theorem 9. Let $i \in\{1,2,3,4\}$ be a fixed number. Suppose that the pseudo-measure $f=\widetilde{f}_{i}$ and real number $\varepsilon>0$ satisfy conditions (6) $-(7)$, and the grid $\widehat{X}$ equals the $\operatorname{grid} X\left(\widetilde{f}_{i}, \varepsilon,|[a, b]|\right)$,

$$
\widehat{X}=X\left(\tilde{f}_{i}, \varepsilon,|[a, b]|\right) .
$$

Then the following statements are right.

1. The number of knots $\widetilde{K}=K\left(\widetilde{f}_{i}, \varepsilon,|[a, b]|\right)$ of this grid satisfies the relations

$$
\begin{gathered}
\sum_{s=0}^{\widetilde{K}-1} \widetilde{f}_{i}\left(\left|\left[x_{s}, x_{s+1}\right]\right|\right) / \varepsilon \leq \widetilde{K}< \\
<\sum_{s=0}^{\widetilde{K}-1} \widetilde{f}_{i}\left(\left|\left[x_{s}, x_{s+1}^{+}\right]\right|\right) / \varepsilon .
\end{gathered}
$$

2. The inequality is true

$$
\left|u_{i}(t)-H_{\widehat{X}} u_{i}(t)\right| \leq \varepsilon \quad \forall t \in|[a, b]|
$$

3. The algorithm for constructing an adaptive grid $\widehat{X}$ has the following properties

$$
N\left(\widetilde{f}_{i}\right)=N_{\text {comp }}\left(\widetilde{f}_{i}\right)=(\widetilde{K}+M+2) .
$$

\section{Digital EXAmples}

As a numerical illustration of the proposed method, several variants of numerical flows generated by functions $u(t)$ in the interval $[0.001, \pi]$. These flows were outraged uniformly distributed on the interval $[0,1]$ a random variable. The purpose of this illustration is to show that the proposed algorithm is very robust against such disturbances. The calculations were carried out using programming in the environment Maple (see [23]). The results are shown in Table 1.

Table 1.

\begin{tabular}{||c|c|c|c|c|c|}
\hline \hline Num & $u(t)$ & $K$ & Compr & Quality & $\begin{array}{c}\text { Time } \\
(\text { sec. })\end{array}$ \\
\hline \hline 1. & $1 / t$ & 7 & 123 & 61.7 & 0.896 \\
\hline 2. & $1 / t$ & 7 & 125 & 62.6 & 0.876 \\
\hline 3. & $\lg t$ & 8 & 111 & 55.7 & 0.921 \\
\hline 4. & $\lg t$ & 8 & 110 & 55.1 & 0.910 \\
\hline 5. & $\sin (t)$ & 757 & 1.32 & 0.661 & 0.876 \\
\hline 6. & $\sin (t)$ & 407 & 2.45 & 1.23 & 0.951 \\
\hline 7. & $\ln t$ & 27 & 35.8 & 17.9 & 0.807 \\
\hline 8. & $\ln t$ & 27 & 35.8 & 17.9 & 0.822 \\
\hline \hline
\end{tabular}

The first column of Table 1 contains the number of the uniformly distributed random flow. The original flow is the sum of the mentioned random flow and the flow of function values $u(t)$ computed on a uniform grid with $M$ nodes $(M=1000)$. The second column contains the function $u(t)$ that generates the mentioned deterministic flow of values on a uniform grid in the segment $[0.001, \pi]$.

In the third column the number of $K$ nodes of the adaptive grid is presented. On the mentioned grid the deviation of the Haar approximation from the function $u(t)$ is at most maximum Haar error when approximating $u(t)$ on the original grid. The mentioned error is precalculated by a special block programs.

The fourth column shows the degree obtained compression (the ratio of the number of nodes of the original grid to the number of nodes of the adaptive grid). The fifth column contains the quality factor of the adaptive compression versus to the standard compression (the standard compression usually is obtained by removing the odd knotes). The last column shows the running time of the program (in seconds).

The obtained results show that compression is very small in the case of slowly changing function. If we are dealing with a rapidly changing function, then the compression is great. Table 1 illustrates the high stability of the proposed adaptive algorithm for random perturbations of the original flow.

\section{Conclusion}

Summing up, we note the main results of this work.

The concept of a pseudo-measure considered here made it possible to construct an adaptive approximation of various probabilistic characteristics of initial flows for random variables. By building the main flows, identifying the main trend is simplified, and the amount of data transmitted through communication channels is significantly reduced.

The new approach considered here differs from the classical wavelet approach by the wide use of irregular grids, which makes it possible to adapt them to the incoming initial flow. This approach is quite effectively applied to the economical selection of the main flow, which determines the main trend of the original random flow.

Several criteria are considered here, among which there are several criteria applicable to the processing of random flows. In particular, the criteria corresponding to the mathematical expectation, dispersion, as well as autocorrelation and cross-correlation of two random flows are considered. It is possible to consider criteria corresponding to various combinations of the mentioned characteristics. The number of knots of the initial (generally speaking, irregular) grid can be arbitrary, and the main grid can be any subset of the initial one. Sequential processing of the initial flow is possible in real time.

\section{REFERENCES}

[1] P. Bloomfield. Fourier analysis of time series: An introduction. New York: Wiley. (1976).ISBN 9780471082569.

[2] R. H. Shumway. Applied statistical time series analysis. Englewood Cliffs, NJ: Prentice Hall. (1988). ISBN 978-0130415004. 
[3] Lin, Jessica; Keogh, Eamonn; Lonardi, Stefano; Chiu, Bill. "A symbolic representation of time series, with implications for streaming algorithms". Proceedings of the 8th ACM SIGMOD workshop on Research issues in data mining and knowledge discovery. New York: ACM Press. pp. 2-11. (2003). CiteSeerX 10.1.1.14.5597. doi:10.1145/882082.882086. S2CID 6084733.

[4] Zhou, Wang, W., Hu, T., Deng, K. Time series forecasting and classification models based on recurrent with attention mechanism and generative adversarial networks//Sensors (Switzerland). 20(24),7211,2020. Pp. 1-20.

[5] E.C. Akrida, G.B. Mertzios, S. Nikoletseas, (...), P.G. Spirakis, V. Zamaraev. How fast can we reach a target vertex in stochastic temporal graphs? Journal of Computer and System Sciences. 114, 2020. Pp. 65-83.

[6] E. Erdemir, P.L. Dragotti, D. Gunduz, PrivacyAware Time-Series Data Sharing with Deep Reinforcement Learning// IEEE Transactions on Information Forensics and Security. 16,9153837,2021. Pp. 389-401

[7] A. Haar. Zur Theorie der orthogonalen Funktionen systeme, Mathematische Annalen, 69, 1910. Pp 331-371. https://link.springer.com/article/10.1007 /BF01456326

[8] S. Mallat. A Wavelet Tour of Signal Processing, Academic Press, 1999. DOI: 10.2118/96553-MS.

[9] Francois Dubeau, Said Elmejdani, Riadh Ksantini. Non-uniform Haar wavelets//Applied Mathematics and Computation. 159, 2004. Pp. 675691. https://www.sciencedirect.com/science/article /abs/pii/S009630030301155X

[10] I.A. Novikov, V. Yu. Protasov, M.A. Skopina. Theory splashes), Moscow: FIZMATGIZ, 2005. 616 p. - ISBN 5-9221-0642-2

[11] Noorazliza Sulaiman, Junita Mohamad-Saleh, Abdul Ghani Abro, Weng-Hooi Tan. An Augmented Bio-Inspired Algorithm (JA-ABC5a) to Design Optimal Digital IIR Filter// WSEAS Transactions on Systems, ISSN / E-ISSN: 1109-2777 / 2224-2678, Volume 19, 2020, Art. \#35, pp. 307-314
[12] Boris Shumilov. Shifted Cubic Spline Wavelets with Two Vanishing Moments on the Interval and a Splitting Algorithm// WSEAS Transactions on Systems, ISSN / E-ISSN: 1109-2777 / 2224-2678, Volume 19, 2020, Art. \#20, Pp. 149-158.

[13] Sang Hoon Lee, Jongsu Park. Novel Branch Prediction Strategy based on Adaptive History Length for High-Performance Microprocessor // WSEAS Transactions on Systems and Control, ISSN / EISSN: 1991-8763 / 2224-2856, Volume 14, 2019, Art. \#43, Pp. 343-351.

[14] Lina A. Shalby. Comparison between Chaos-Control Methods Efficiency for Discrete Systems// WSEAS Transactions on Systems and Control, ISSN / EISSN: 1991-8763 / 2224-2856, Volume 14, 2019, Art. \#36, pp. 284-290

[15] Saugata Dutta, Kavita Saini. Statistical Assessment of Hybrid Blockchain for SME Sector //WSEAS Transactions on Systems and Control, ISSN / EISSN: 1991-8763 / 2224-2856, Volume 16, 2021, Art. \#6, pp. 83-95

[16] Yu.K. Demyanovich. Spline waveform theory. - SPb $\therefore$ Publishing house of St. Petersburg. University, 2013. 526 p. ISBN 978-5-288-05505-8

[17] Yu. K. Demyanovich, A.Yu. Ponomareva. "Adaptive Spline-Wavelet Processing of a Discrete Flow" // J. Math. Sci., New York 210, No 4, 2015. Pp.371-390. DOI: 10.1007/s10958-015-2571-6.

[18] H. Saxena, A. Singh, J.N. Rai. Adaptive splinebased PLL for synchronisation and power quality improvement in distribution system. IET Generation, Transmission and Distribution, 14 (7). 2020. Pp. 1311-1319.

[19] D. Cornel, A. Buttinger-Kreuzhuber, A. Konev, Z. Horvth, M. Wimmer, R. Heidrich, J. Waser. Interactive visualization of flood and heavy rain simulations. Computer Graphics Forum, 38 (3). 2019. Pp. 25-29.

[20] Z. Yang, H. Liu, T. Bi, Z. Li, Q. Yang. An adaptive PMU missing data recovery method. International Journal of Electrical Power and Energy Systems, 116. 2020. Pp. 505-537.

[21] I.G. Burova, E.F. Muzafarova, D.E. Zhilin. About adaptive grids construction. WSEAS Transactions on Mathematics, 17. 2018. pp.340-351.

[22] Digital Signal Processing Application Using the ADSP-2100 Family. - Prentice Hall, Englwood Cliffs, 1992.

[23] [40] Maple 2017.0, Product Build IDs, Maple Build ID 1231047, Licensed to: Prof. Yuri Demyanovich, Serial Number: M4SUJR24AKMC7YDY, Permanent Licence.

\section{Creative Commons Attribution License 4.0} (Attribution 4.0 International, CC BY 4.0)

This article is published under the terms of the Creative Commons Attribution License 4.0 https://creativecommons.org/licenses/by/4.0/deed.en_US 